[0212-7199 (2005) 22: 3; pp 114-117] ANALES DE MEDICINA INTERNA Copyright (C) 2005 ARAN EDICIONES, S.L.

AN. MED. INTERNA (Madrid) Vol. 22, N. ${ }^{\circ}$ 3, pp. 114-117, 2005

\section{Manifestaciones satélites de las enfermedades internas}

\author{
E. PORTUGAL FERNÁNDEZ DEL RIVERO' ${ }^{1}$, J. PORTUGAL ÁLVAREZ², \\ A. DEL CASTILLO RUEDA ${ }^{2}$, C. RECARTE GARCÍA-ANDRADE², \\ M. D. CASAS GONZÁLEZ
}

${ }^{1}$ Servicio de Psiquiatría. Hospital General Universitario Gregorio Marañón. Madrid. Centro de Salud Mental de Vilanova y la Geltru. Barcelona. ${ }^{2}$ Servicio de Medicina Interna II. Hospital General Universitario Gregorio Marañón. Madrid

SATELLITE MANISFESTATIONS OF INTERNAL DISEASES

\section{RESUMEN}

Objetivos. Definir el concepto de manifestaciones satélites (MS) y determinar que número y tipos de ellas aparecen en las enfermedades de cada una de las subespecialidades de la Medicina Interna.

Método: Se revisan 290 enfermedades en tratados de Medicina Interna y de especialidades y en cada una de ellas se describen los tipos de MS que presentan.

Resultados: Las enfermedades respiratorias son las que mas MS presentan y las que menos las neurológicas. El tipo mas frecuente de MS es dermatológico. Las enfermedades que más variedad tienen de tipos de MS son las endocrinas. Analizando los porcentajes de cada tipo de MS en relación con el número de enfermedades de cada grupo, los mas altos son de MS dermatológicas en enfermedades cardiacas.

Conclusiones: Las MS son un fenómeno muy frecuente en la clínica médica actual y expresan el carácter holístico de la enfermedad. Su conocimiento es imprescindible en la clínica internista y subespecializada, donde pueden suponer una ayuda diagnostica, aunque un mal conocimiento de su realidad pueden significar lo contrario.

PALABRAS CLAVE: Manifestaciones satélites. Medicina Interna.

\begin{abstract}
Aims: Define the concept of satellite manifestations (SM) and to determine the number and types of them that appear in the diseases of each one of the subspecialities of the Internal Medicine.

Methods: 290 diseases are reviewed in agreements of Internal Medicine and specialities books and in each one are described the types of SM that they present.

Results: The respiratory diseases are the group that more SM presents and the less one the neurological. The most frequent type of SM are dermatological. The diseases that have more variety of types of SM are the endocrinologicals. Analyzing the percentages of every type of SM in relation with the number of diseases of every group, the highest are the dermatological SM in cardiac diseases.

Conclusions: The SM are a very frequent phenomenon in the current medical practice and express the holistic character of the disease. His knowledge is indispensable in the internal medicine and subspecialities, where they can suppose a help in the diagnoses, though a bad knowledge of his reality can mean the opposite.
\end{abstract}

KEY WORDS: Satellite manifestations. Internal Medicine.

Portugal Fernández del Rivero E, Portugal Álvarez J, Del Castillo Rueda A, Recarte García-Andrade C, Casas González. MD. Manifestaciones satélites de las enfermedades internas. An Med Interna (Madrid) 2005; 22: 114-117.

\section{INTRODUCCIÓN}

Entendemos por manifestaciones satélites (MS) de una enfermedad, aquellas que aparecen en forma de síntomas y signos y que no son propias del órgano, aparato o sistema que constituyen la base de la enfermedad. El proceso morboso afecta por lo común a una estructura organizada de la economía corporal (órgano, aparato, sistema) y a partir de ella aparecen las manifestaciones propias que han permitido la descripción característica de la enfermedad. La realidad de enfermedad como entidad clínica se ha constituido sobre la base de la especial conjunción de síntomas y signos que obliga a explicaciones etiológicas, patogénicas y fisiopatológicas con el referendo anatomopatológico. Con estas entidades clínicas se ha constituido toda la Nosología Médica obedeciendo no tanto a la estricta realidad biológica como a la tendencia taxonómica de la mente humana y a facilitar el conocimiento y manejo de las enfermedades. Así cada enfermedad (cardiaca, hepática, pulmonar, cerebral, etc.) tiene sus manifestaciones, más o menos sensibles y específicas, que le son propias y que configuran el cuadro clínico. Pero en ocasiones se asocian manifestaciones en órganos, aparatos o sistemas distintos de los que constituyen la base anatómica de la enfermedad, por ejemplo, manifestaciones

Trabajo aceptado: 22 de noviembre de 2004

Correspondencia: J. de Portugal Álvarez. Servicio de Medicina Interna II. Hospital General Universitario Gregorio Marañón. C/ Doctor Esquerdo, 46. 28007Madrid 
cardíacas en las enfermedades pulmonares, manifestaciones digestivas en las enfermedades del riñón o neurológicas en las endocrinas, etc. Estas manifestaciones satélites, en las cuales subyace el carácter holístico de la enfermedad, se realizan por mecanismos diversos (etiológicos, patogénicos, fisiopatológicos, etc.) y tiene su importancia en la clínica según el saber y la habilidad del médico.

En este trabajo se revisan la mayor parte de las enfermedades de la Medicina Interna y en cada una de ella se identifican las diversas manifestaciones satélites discutiendo su relevancia y sus posibilidades de manejo práctico.

\section{MATERIAL Y MÉTODO}

Se han revisado 290 enfermedades de Medicina Interna en una reciente edición de un tratado de medicina (1). Se ha utilizado también un libro especialmente dedicado a las MS (2) y ocasionalmente se han consultado textos de las distintas subespecialidades de la Medicina Interna (3-10). La metódica de este trabajo hace que las referencias bibliográficas se limiten a libros cuya consulta ha sido completa, por lo que en las referencias no se especifican páginas. En cada enfermedad de los distintos aparatos y sistemas se han buscado las MS utilizando los siguientes criterios: a) se consideran MS de un enfermedad los síntomas y signos que aparecen en órganos, aparatos o sistemas distintos de los que constituyen la base fundamental de la enfermedad y se integran, con mayor o menor categoría, en el cuadro clínico; b) se han valorado las MS de una enfermedad cuando su aparición en ésta es constante o frecuente, prescindiendo por tanto de aquellas de expresión ocasional y poco frecuente. Tal discriminación se basa en criterios clínicos propios, las descripciones del texto analizado y, eventualmente, en la consulta de textos especializados; c) no se valoran en nuestro trabajo como MS datos hematológicos, bioquímicos, inmunológicos, radiológicos, histológicos, etc., aunque ocasionalmente también pueden tener categoría satélite; d) no se han considerado las enfermedades sistémicas y básicamente generalizadas que carecen de localización concreta y característica y tienen manifestaciones múltiples diseminadas en el organismo. Es el caso de las conectivopatías, vasculitis, enfermedades metabólicas, infecciosas, etc.; e) se ha procurado no incluir como MS las "enfermedades asociadas" considerando como tales aquellas entidades con categoría completa de enfermedad (no simples síntomas y signos) que se asocian con significación estadística a la enfermedad probando y cuyos mecanismos de asociación suelen conocerse, aunque no siempre; y f) también se ha prescindido del análisis de síndromes, salvo los casos en que estén ligados a una causa concreta del organismo y no sean un concepto general que incluya enfermedades especiales.

De cada grupo de enfermedades se obtiene: a) número total de MS en cada grupo de enfermedades; b) tipos distintos de MS en el conjunto total de enfermedades analizadas; c) número de tipos distintos de MS en cada grupo de enfermedades; d) porcentaje de cada tipo de MS en relación con el número de enfermedades de cada grupo; y e) posteriormente se realiza la misma metódica en otro tratado de Medicina Interna (11) de principios del siglo pasado, con el fin de apreciar en el tiempo la valoración de las MS.

\section{RESULTADOS}

En la tabla I, realizada mediante datos obtenidos básicamente de (1), se exponen resultados que pueden expresarse en los siguientes apartados:

1. Número total de MS en cada grupo, es decir veces que los tipos de MS, repetidos o no, aparecen en cada grupo. Las enfermedades respiratorias son las que más MS presentan $(n=169)$, seguidas de las neurológicas $(n=153)$ y endocrinas $(\mathrm{n}=147)$. Las que menos MS en total presentan son las nefrológicas $(n=75)$ y las digestivas $(n=81)$.

2. Tipos distintos de MS en el conjunto total de enfermedades $(\mathrm{n}=290)$. Las más frecuentes son las dermatológicas $(n=141)$ y las menos frecuentes las hepáticas $(n=24)$ y las nefrológicas $(\mathrm{n}=38)$.

3. Número de tipos distintos de MS en cada grupo de enfermedades. Las enfermedades que más variedad tienen de tipos de MS son las endocrinas $(n=109)$ y las neurológicas $(n=102)$ y las que menos las digestivas $(n=54)$, las nefrológicas $(n=70)$ y las hepáticas $(\mathrm{N}=72)$.

4.- Si analizamos el porcentaje de cada tipo de MS en relación con el número de enfermedades de cada grupo encontramos que los porcentajes más altos son de MS dermatológicas en enfermedades cardíacas $(96,6 \%)$, las de MS digestivas en enfermedades respiratorias $(87 \%)$, y las MS digestivas en hepatopatías $(79,2 \%)$. En cambio los porcentajes más bajos se encuentran en MS endocrinas en enfermedades músculoesqueléticas (10\%), en MS nefrológicas en enfermedades neurológicas $(14,3 \%)$ y MS hepáticas en enfermedades respiratorias $(14,6 \%)$.

5. En la tabla II obtenida del número de enfermedades de cada aparato (11) es claramente inferior a los recogidos en la tabla I y esto influye en que el número de MS de cada aparato y el número de variedades de MS en cada tipo de enfermedad, también sean menores. Otros factores que después comentaremos pueden influir en estas diferencias.

\section{DISCUSIÓN}

Las MS, en el sentido que las hemos descrito aquí, suponen un fenómeno muy frecuente en la clínica médica actual: en la patología de todos los aparatos y sistemas existen MS si bien en variedad y proporción diversas. Las enfermedades que más variedad presentan de MS son las endocrinas y las neurológicas y también el mayor número total de MS, si bien son los grupos que más enfermedades contienen, aunque la relación entre número total de MS y el de enfermedades no es significativamente superior al de la mayoría de los tipos de MS que en los otros aparatos y sistemas. Es decir todos los grupos de enfermedades tienen semejante proporción y distribución de MS.

Esta alta incidencia de MS en las enfermedades médicas de la clínica actual es consecuencia del mejor conocimiento fisiopatológico y anatomopatológico de las enfermedades, lo cual parece ponerse de manifiesto en el menor número de MS en las descripciones clínicas de hace cien años, cuando dichos conocimientos eran muy insuficientes. En cualquier caso, el fenómeno de las MS es una expresión clínica del carácter holístico de la enfermedad y bajo esta conceptuación deben ser valoradas por el médico, obligado a un conocimiento lo más amplio posible de la patología. Esto debe ser especial- 
TABLA I

TIPOS DE MANIFESTACIONES SATÉLITES

Crupos de Cardiacas Respiratorias Digestivas Hepáticas Dermatológicas Músculo esquel. Neurológicas Nefrológicas Endocrinológ. Oftalmológicas Total \begin{tabular}{lllllllllllllllllllllllllllllllllll} 
enfermedades & $(n)$ & $A$ & $B$ & $\%$ & $A$ & $B$ & $\%$ & $A$ & $B$ & $\%$ & $A$ & $B$ & $\%$ & $A$ & $B$ & $\%$ & $A$ & $B$ & $\%$ & $A$ & $B$ & $\%$ & $A$ & $B$ & $\%$ & $A$ & $B$ & $\%$ & $A$ & $B$ & $\%$ & $A$ & $B$ \\
\hline Cardiacas & 29 & & & 7 & 24 & 82,8 & 12 & 18 & 62,1 & 4 & 9 & 31,0 & 17 & 28 & 96,6 & 9 & 17 & 58,6 & 11 & 21 & 72,4 & 2 & 7 & 24,1 & 2 & 5 & 17,2 & 11 & 14 & 48,3 & 75 & 143
\end{tabular} Respiratorias $\begin{array}{lllll}14 & 7 & 13 & 92,9\end{array}$ $91741,53 \quad 6 \quad 14,617 \quad 3175,6 \quad 4 \quad 19135,712 \quad 36257,1$

$514100,0416114,368169$

$\begin{array}{lllllllll}\text { Digestivas } & 43 & 4 & 10 & 23,3 & 5 & 19 & 44,2\end{array}$

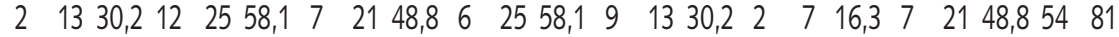

Hepáticas $\quad \begin{array}{llllllllll}24 & 6 & 8 & 33,3 & 4 & 6 & 25,0 & 4 & 19 & 79,2\end{array}$

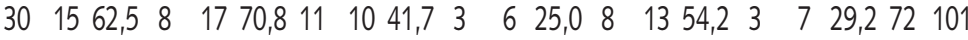
Músc. esquel. $\begin{array}{llllllllllllllll}30 & 11 & 10 & 33,3 & 6 & 12 & 40,0 & 6 & 9 & 30,0 & 3 & 7 & 23,3 & 23 & 13 & 43,3\end{array}$ $13 \quad 15 \begin{array}{llllllllllll}50,0 & 8 & 9 & 30,0 & 2 & 3 & 10,0 & 20 & 17 & 56,7 & 92 & 95\end{array}$

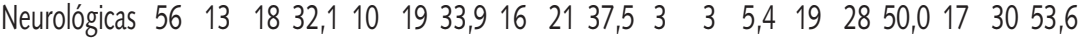
$\begin{array}{llllllllll}7 & 8 & 14,3 & 11 & 14 & 25,0 & 15 & 12 & 21,4102153\end{array}$

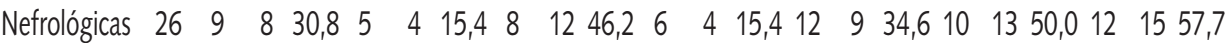
$\begin{array}{llllllll}3 & 5 & 19,2 & 5 & 5 & 19,2 & 70 & 75\end{array}$

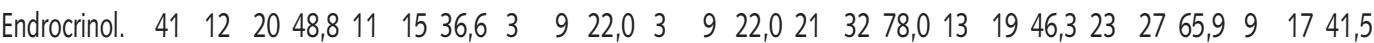
111126,8109147 \begin{tabular}{lllllllllllllllllllllllllllllllll}
\hline TOTAL & 290 & 69 & 104 & 35,9 & 48 & 99 & 34,1 & 68 & 117 & 40,3 & 24 & 51 & 17,6141 & 181 & 62,4 & 68 & 136 & 46,9 & 88 & 149 & 51,4 & 38 & 59 & 20,3 & 33 & 61 & 21,0 & 76 & 103 & 35,5
\end{tabular}

A: número de tipos distintos de manifestaciones satélites

$\mathrm{B}$ : número total de manifestaciones satélites y \% en relación con el número de enfermedades

\section{TABLA II}

\section{TIPOS DE MANIFESTACIONES SATÉLITES}

Grupos de Cardiacas Respiratorias Digestivas Hepáticas Dermatológicas Músculo esquel. Neurológicas Nefrológicas Endocrinológ. Oftalmológicas Total

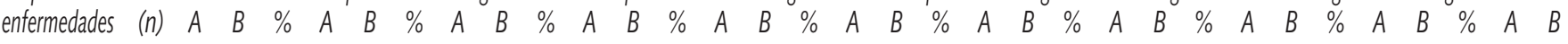

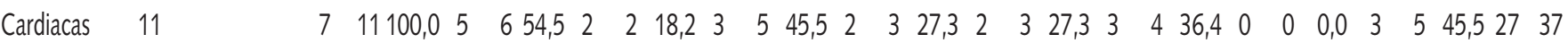
$\begin{array}{llllllllllllllllllllllllllllllll}\text { Respiratorias } & 14 & 7 & 13 & 92,9 & 0,0 & 2 & 5 & 35,7 & 0 & 0 & 0,0 & 2 & 4 & 28,6 & 1 & 3 & 21,4 & 3 & 5 & 35,7 & 1 & 3 & 21,4 & 0 & 0 & 0,0 & 1 & 2 & 14,3 & 17 & 35\end{array}$ $\begin{array}{llllllllllllllllllllllllllllllll}\text { Digestivas } & 23 & 6 & 11 & 47,8 & 0 & 0 & 0,0 & 0,0 & 2 & 4 & 17,4 & 0 & 0 & 0,0 & 0 & 0 & 0,0 & 0 & 0 & 0,0 & 0 & 0 & 0,0 & 0 & 0 & 0,0 & 0 & 0 & 0,0 & 8 & 15\end{array}$ $\begin{array}{lllllllllllllllllllllllllllllllll}\text { Hepáticas } & 11 & 1 & 2 & 18,2 & 0 & 0 & 0,0 & 5 & 8 & 72,7 & 0,0 & 7 & 7 & 63,6 & 1 & 2 & 18,2 & 5 & 5 & 45,5 & 3 & 3 & 27,3 & 1 & 1 & 9,1 & 0 & 0 & 0,0 & 24 & 28\end{array}$

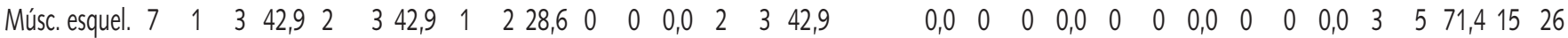

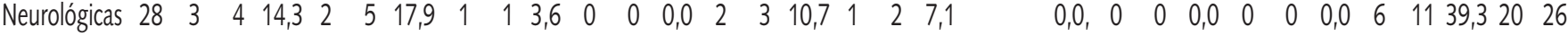

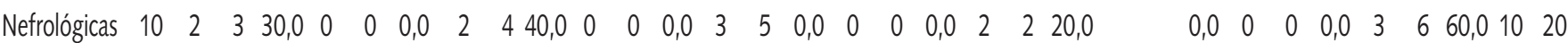
$\begin{array}{lllllllllllllllllllllllllllllllll}\text { Endrocrinol. } & 6 & 1 & 2 & 33,3 & 0 & 0 & 0,0 & 2 & 2 & 33,3 & 0 & 0 & 0,0 & 2 & 4 & 66,7 & 1 & 3 & 50,0 & 3 & 4 & 66,7 & 0 & 0 & 0,0 & & 0,0 & 3 & 4 & 66,7 & 11 & 19\end{array}$ $\begin{array}{lllllllllllllllllllllllllllllllll}\text { TOTAL } & 110 & 21 & 38 & 34,6 & 11 & 18 & 16,3 & 18 & 28 & 25,4 & 4 & 6 & 5,4 & 25 & 39 & 35,5 & 6 & 13 & 11,8 & 15 & 19 & 17,3 & 9 & 10 & 9,1 & 1 & 1 & 0,9 & 19 & 33 & 30,0\end{array}$

A: número de tipos distintos de manifestaciones satélites

B: número total de manifestaciones satélites y \% en relación con el número de enfermedades

mente sugestivo para el especialista y el subespecialista que tienen el riesgo de encerrarse en sus parcelas clínicas y no valorar convenientemente la dimensión holística de la enfermedad. Este riesgo, que emana de un insuficiente conocimiento de la enfermedad, puede abocar a una defectuosa práctica de su especialidad, haciendo verosímil la paráfrasis de un viejo adagio letamendiano: "el especialista que sólo sabe de su especialidad ni de su especialidad sabe".
Generalmente las MS tienen una relevancia clínica menor que los síntomas y signos propios o clásicos de la enfermedad, pero no siempre es así y pueden ser especialmente importantes y sugerentes para el diagnóstico, incluso cuando constituyen la primera señal de la enfermedad antes de que ésta haya expresado su cuadro característico. Por lo tanto, para el médico avisado pueden suponer una ayuda en su pesquisa diagnóstica, si bien en ocasiones son capaces de suscitar la duda y complicar dicha 
pesquisa. Pero el médico que conozca mal las MS, incluso su fundamento fisiopatológico, la frecuencia y la dimensión de la duda serán mayores y también la posibilidad de error.

La comparación de datos referentes a las MS en un tratado de Medicina de 1919 (cuya primera edición es de 1883) con los de un tratado actual ofrecen pocas posibilidades de resultados valorables. En principio, el número de enfermedades de cada aparato o sistema es sensiblemente menor en el tratado antiguo, posiblemente porque muchas de ellas no habían sido identificadas, se encuentran englobadas en grandes síndromes o son integradas como formas clínicas en una misma enfermedad. Por otra parte, en el tratado de 1919 el número total y el de variedades de MS son también menores que en el tratado actual, bien porque fueran desconocidas o no se identificaran y valoraran como tales, bien, como ocurre en alguna ocasión, porque son interpretadas con entidad propia y autónoma del

\section{Bibliografía}

1. Braunwald E, Fauci AS, Kasper DL, et al. Harrison's Principles of Internal Medicine (15th edit) McGraw Hill 2001.

2. Portugal Álvarez J. Manifestaciones Satélites de la enfermedades internas. Masson 1993.

3. De Groot CJ, Jameson JL Ed. Endocrinology Saunders Co. 14th ed. 2001.

4. Ruddy Sh, Harris ED, Sludge CB. Textbook of Rheumathology. Saunders Co. 6th ed 2001.

5. Schiff EK, Sorrel MF, Maddrey WC. Diseases of the Liver. Ed Lippincott Williams and Wilkins. 9th edition 2002.

6. Fraser RS, Müller NL, Colman N Paré PA Diagnosis of diseases of the aparato donde se expresan. La realidad es que en varios grupos de enfermedades no se aprecian uno o varios grupos de MS, siendo llamativo que en el grupo de 23 enfermedades digestivas sólo se encuentran manifestaciones cardíacas y hepáticas como satélites. No deja de llamar la atención esta relativa pobreza de MS en un tratado que pertenece a una época en que las descripciones clínicas habían adquirido una extensión y finura extraordinarias. Quizá la explicación esté en el insuficiente conocimiento fisiopatológico de los fenómenos clínicos que, consecuentemente, daba más importancia a la descripción del fenómeno que a su explicación. Esta evolución en el tiempo de las MS expresa una vez más el valor del pensamiento fisiopatológico en la interpretación de los acontecimientos clínicos e incita a someter a ese conocimiento la valoración de este tipo de manifestaciones, por otra parte siempre aconsejable en la actividad clínica. chest. Saunders Co. 4th ed 1999.

7. Braunwald E, Zipes DP, Libby P. Heart Diseases Saunders Co. 6th ed 2001.

8. Bradley WG, Daroff RB, Femichel GM, Manden CD. Neurology in Clinical Practice. Butterworth Heinemann. 3th ed 2000.

9. Yamada T, Alpers DH Laine L, et al. Textbook of Gastroenterology. Lippincott Williams and Wilkins 3th ed 1999.

10. Brenner BM, Brenner and Rector's The Kidney. Saunders Co. 7th ed 2004.

11. Strümpell, A. Patología y terapéutica de las enfermedades internas. Seix Edit. (21 $1^{\mathrm{a}}$ ed) 1919. 